\title{
Shifted unitary orthogonal methods for the overlap inversion
}

\author{
Artan Boriçi*† \\ Physics Department \\ University of Tirana \\ Blvd. King Zog I \\ Tirana - Albania \\ E-mail: borici@fshn.edu.al
}

\author{
Alban Allkoçi \\ Computer Science Section \\ Polytechnic University of Tirana \\ Mother Theresa Square \\ Tirana - Albania \\ E-mail: albandfie.upt.al
}

\begin{abstract}
In this work we compare the convergence of the shifted unitary orthogonal method (SUOM) and different Krylov subspace solvers for propagator computations with overlap fermions. We show that the SUOM algorithm performs similarly to the shifted unitary minimal residual method (SUMR) with the latter converging slightly faster. When the geometric optimality is applied to SUOM we get e new algorithm which is faster than SUMR.
\end{abstract}

XXIIIrd International Symposium on Lattice Field Theory

25-30 July 2005

Trinity College, Dublin, Ireland

\footnotetext{
* Speaker.

The authors participation in the conference and part of this work are funded from the NATO grant EAP.RIG.981410.
} 


\section{Introduction}

In this paper we report on the progress that we have made in the search for an optimal Krylov subspace method for overlap fermions. In the previous lattice conference we reported preliminary results on a new method, the shifted unitary orthogonal method or SUOM [1]. The method is in fact the three-term-recurrence specialisation of the full orthogonalisation method (FOM) in case of shifted unitary matrices. Likewise, the shifted unitary minimal residual method (SUMR), discovered earlier by [2, 3] is a short-recurrence specialisation of the generalised minimal residual method (GMRES).

Our task is to solve the linear system:

$$
D x=b, \quad x, b \in \mathbb{C}^{N}, \quad D \in \mathbb{C}^{N \times N}
$$

where $D$ is the Neuberger's Overlap operator [Ð⿻:

$$
D=c_{1} I+c_{2} V
$$

Here $c_{1}=(1+m) / 2, c_{2}=(1-m) / 2$ and $m$ are the bare fermion mass, $V$ is a unitary matrix given by:

$$
V=D_{W}\left(D_{W}^{*} D_{W}\right)^{-\frac{1}{2}}
$$

where $D_{W}$ is Wilson-Dirac lattice operator.

\section{Arnoldi iteration for unitary matrices}

Some time ago Rutishauser [5] observed that for upper Hessenberg unitary matrices one can write $H=L U^{-1}$, where $L$ and $U$ are lower and upper bidiagonal matrices. Applying this decomposition for the Arnoldi iteration,

$$
V Q_{k}=Q_{k} H_{k}+h_{k+1, k} q_{k+1} e_{k}^{T}
$$

one obtains:

$$
V Q_{k} U_{k}=Q_{k} L_{k}+h_{k+1, k} q_{k+1} e_{k}^{T} .
$$

Since $U_{k}, L_{k}$ are bidiagonal matrices we arrive to the following three-term recursion:

$$
l_{k+1, k} q_{k+1}=V q_{k}-q_{k} l_{k k}+V q_{k-1} u_{k-1, k}
$$

This way we obtain the unitary Arnoldi process shown in Algorithm 1. If we denote $\tilde{H}_{k}=H_{k}+$ $l_{k+1, k} e_{k} e_{k}^{T}$, it is easy to show that for the unitary Arnoldi process we get:

$$
\tilde{H}_{k}^{*} \tilde{H}_{k}=H_{k}^{*} H_{k}+l_{k+1, k}^{2} e_{k} e_{k}^{T}=I_{k}
$$




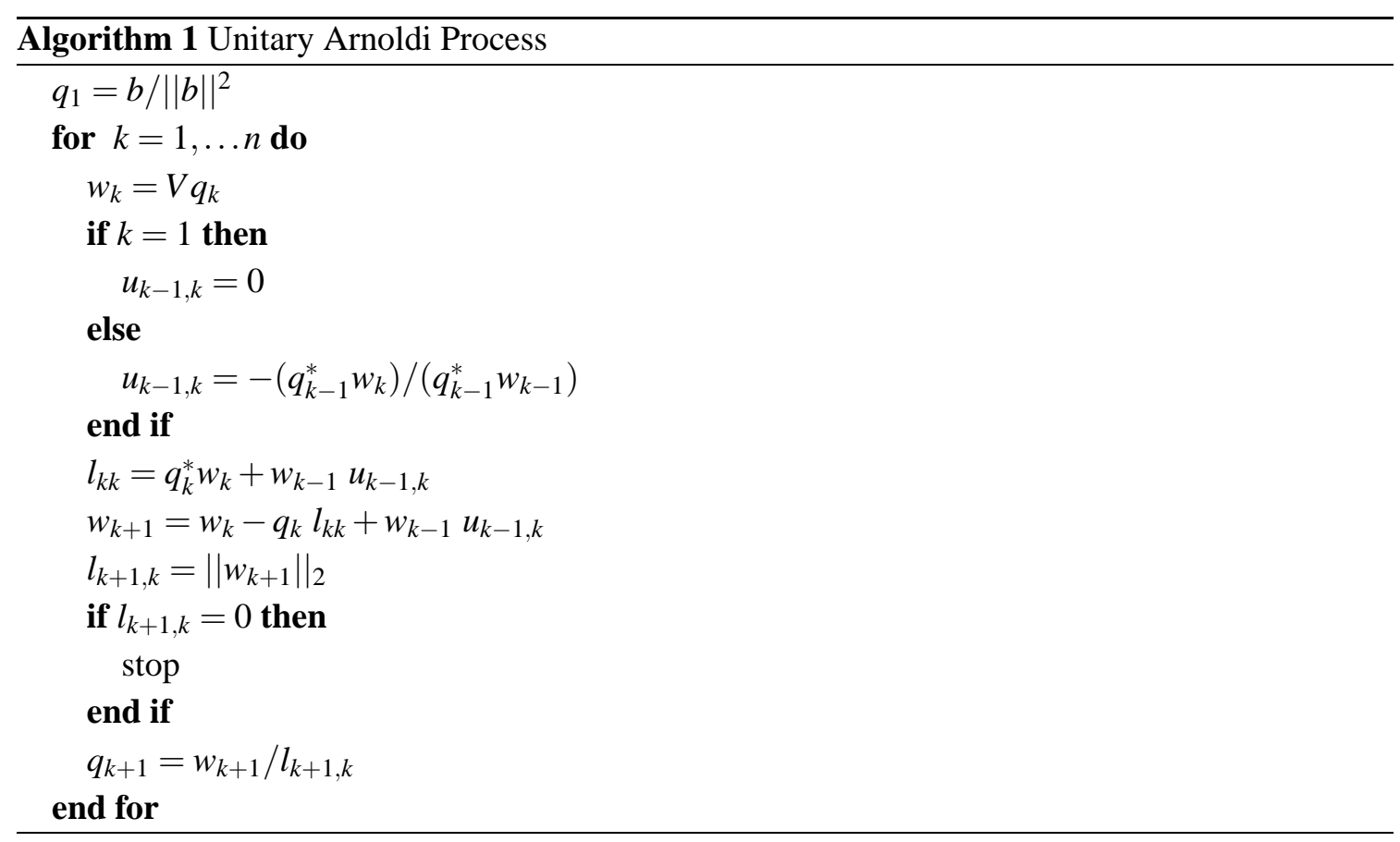

\section{The SUOM algorithm}

Using the unitary Arnoldi process one can ask an approximate solution of 1.1 as a linear combination of the Arnoldi vectors $Q_{k}$. This leads to solving the smaller linear system:

$$
\left(c_{1} I_{k}+c_{2} L_{k} U_{k}^{-1}\right) y_{k}=e_{1}
$$

The matrix in the left hand side is upper Hessenberg. In order to solve a simpler system we define $z_{k}=U_{k}^{-1} y_{k}$ and get:

$$
\left(c_{1} U_{k}+c_{2} L_{k}\right) z_{k}=e_{1}
$$

where now $T_{k}=c_{1} U_{k}+c_{2} L_{k}$ is a tridiagonal matrix. Note that the solution to the above system can be updated recursively and this way one can update the solution of the original system by short recurrences. Here we omit the details and refer the interested reader to a forthcoming publication [6]. The resulting algorithm is shown in Algorithm 2. Note that $D w_{k}$ multiplication is redundant. Indeed multiplying by $D$ both sides of the equation:

$$
w_{k}=q_{k}+u_{k-1 k} q_{k-1}-c_{1} u_{k-1 k} / \tilde{l}_{k-1 k-1} w_{k-1}
$$

and saving the $V q_{k}$ and $V q_{k-1}$ vectors one obtains for free the $D w_{k}$ vector.

The SUOM algorithm is algebraically optimal. This means that the algorithm constructs a new residual vector which is orthogonal to the Krylov subspace already in place. However, a more satisfying optimality is the geometric optimality, which is a feature of algorithms with a new residual being smaller than the previous one. In this case one seeks the solution such that the residual vector norm is minimal:

$$
x=\arg \min \|b-D x\|_{2}
$$




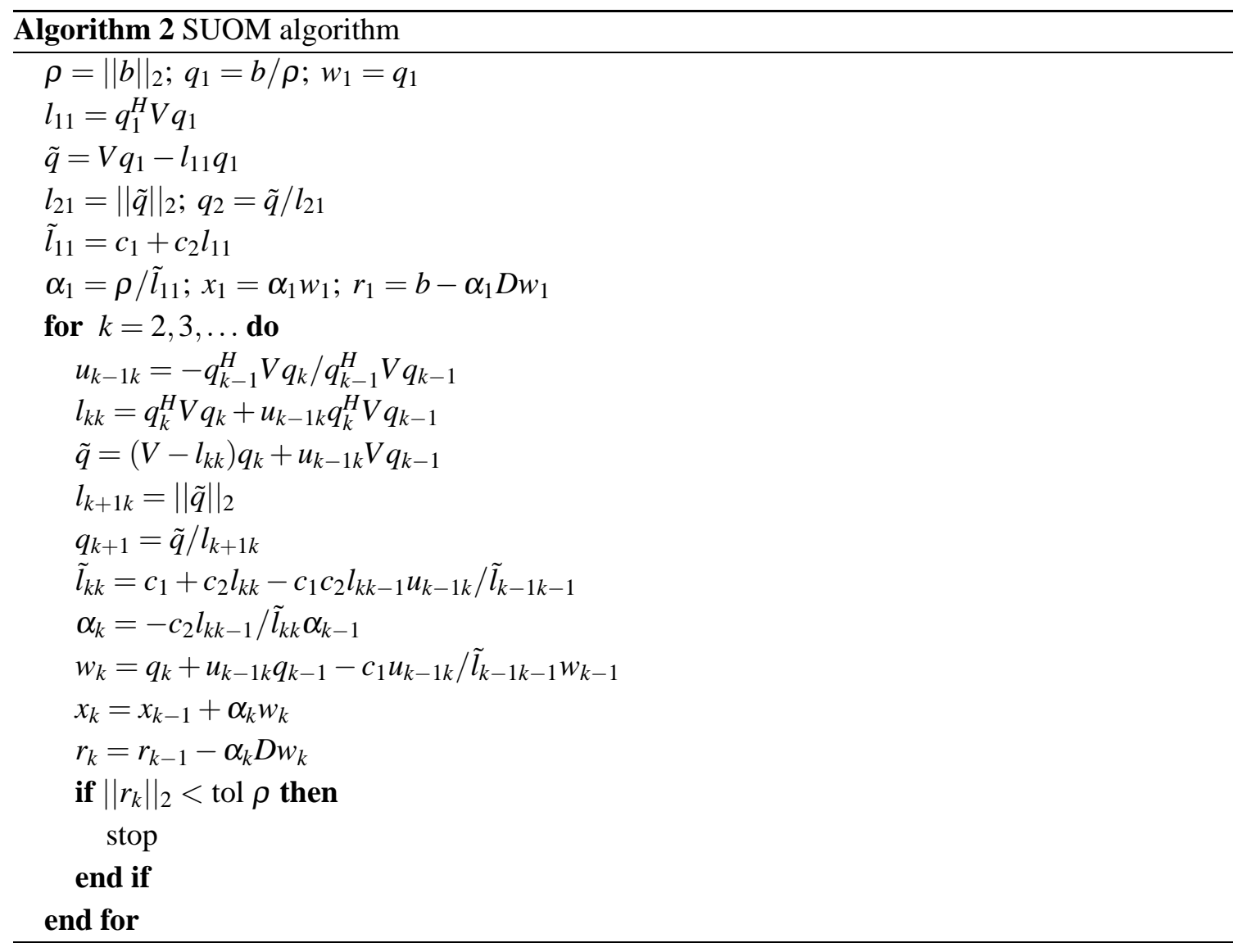

Arnoldi process offers an orthogonal basis vectors which can be used to project the above large least squares problem into a smaller problem:

$$
\tilde{y}_{k}=\arg \min \left\|e_{1}-\tilde{H}_{k} \tilde{y}_{k}\right\|_{2}
$$

The algorithm that is derived this way is a minimal residual algorithm for shifted unitary matrices. We call it SUOM+ since it is a different algorithm from SUMR. The latter uses Givens rotations to implement the isometric Arnoldi process [2] mentation used in our case, Algorithm 1. The full details of the SUOM+ algorithm can be found in [6].

\section{Comparison of algorithms}

In Figure 1 we show the convergence of various algorithms as a function of Wilson matrixvector multiplication number on $8^{3} 16$ lattices at various couplings and quark masses. For the overlap matrix-vector multiplication we use the double pass Lanczos algorithm (without small eigenspace projection of $H_{W}$ )

We show the convergence os SUOM, SUMR, Conjugate Residuals (CR), Conjugate Gradients on Normal Equations (CGNE) and CG-CHI. The latter is the CGNE which solves simultaneously the decoupled chiral systems appearing in the matrix $D^{*} D$. We have preliminary results for the $\mathrm{SUOM}+$ algorithm in one case only. 

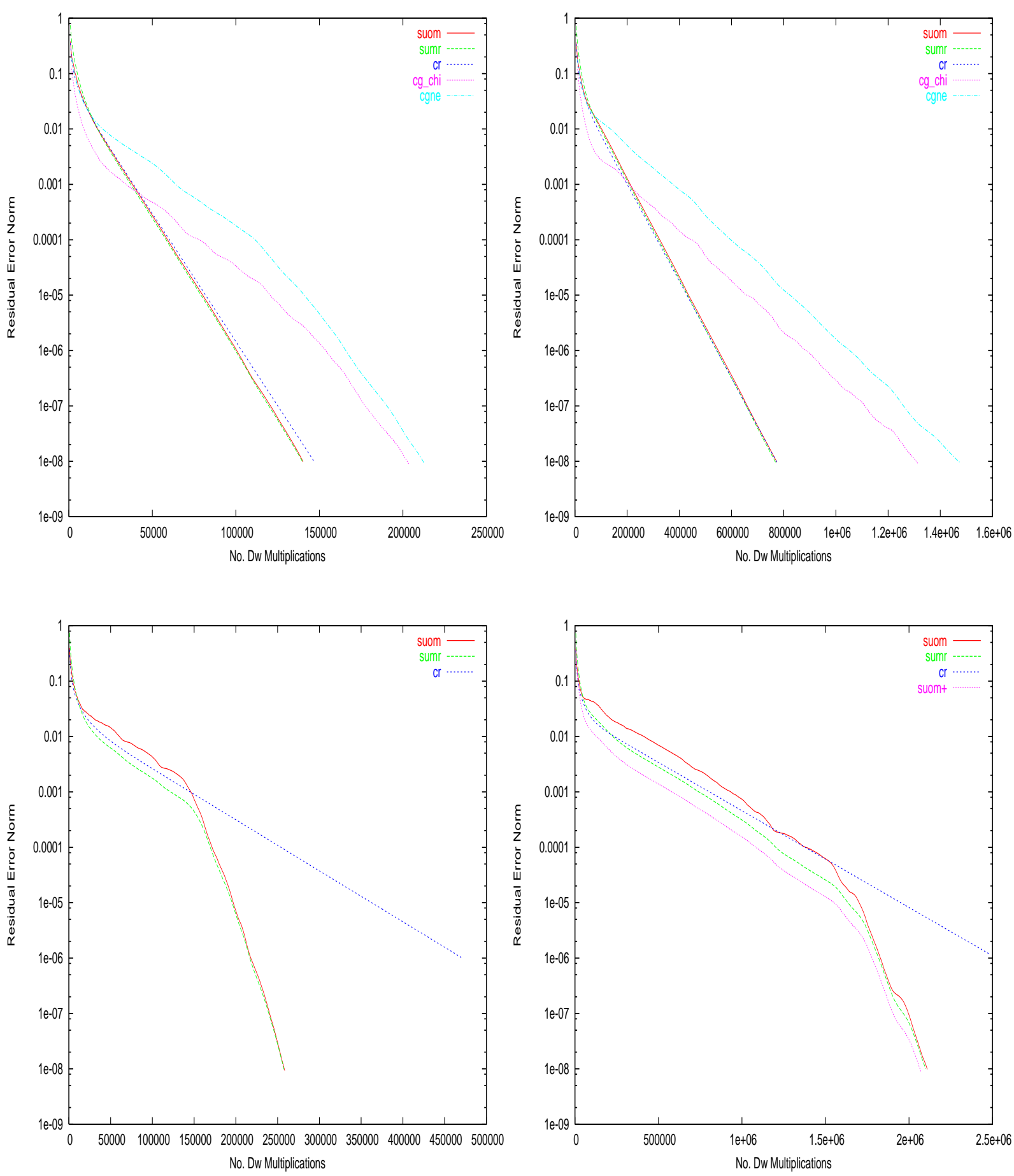

Figure 1: Convergence history of various solvers for quark masses $m=0.05$ (upper panel) and $m=0.01$ (lower panel) on background gauge fields at $\beta=6$ (left panel) and $\beta=5.7$ (right panel).

The first observation is that for quark propagator computations SUOM, SUMR and CR are more efficient than CGNE and CG-CHI algorithms. This is observed by the other groups as well [3]. This is why we did not run further these algorithms for smaller quark masses. 
Another interesting observation is that CR converges neck-to-neck with SUOM and SUMR algorithms for moderate quark masses. As this is lowered, we see that SUOM and SUMR convergence rate becomes larger than that of $\mathrm{CR}$ at a certain accuracy threshold, which depends on $\beta$.

Hence, the best algorithms are the optimal algorithms SUOM and SUMR. These converge in all cases similarly with SUMR being slightly faster, something which should be expected from the geometric optimality of SUMR.

However, we see, in the only case available, that SUOM+ converges faster than SUOM and SUMR. We expected a different behaviour of SUOM+ from SUMR, but there are no theoretical grounds to expect that SUOM+ is faster than SUMR. Since the result is preliminary, further tests are needed to make a definite conclusion.

\section{Conclusion}

We have shown how to build a class of optimal iterative solvers for overlap fermions using the unitary Arnoldi process 1. This is easier to implement using the Rutishauser decomposition [5] than Givens rotations [2].

Preliminary results show that SUOM+ algorithm may converge faster then SUMR. It is expected that different implementations can give different results and in our case this is obvious. What is not obviuos is which implementation is faster and this has to be further investigated [6].

\section{References}

[1] A. Boriçi, hep-lat/0409078.

[2] C. F. Jagels und L. Reichel, Num. Lin. Algeb. Appl., Vol. 1(6), 555-570 (1994)

[3] G. Arnold et al, hep-lat/0311025

[4] H. Neuberger, Phys. Lett. B 417 (1998) 141

[5] H. Rutishauser, Numer. Math. 9 (1966) 104

[6] A. Allkoçi and A. Boriçi, in preparation. 